\title{
Evaluating the Value of the Respiratory Therapist: Where Is the Evidence? Focus on the Barnes-Jewish Hospital Experience
}

\author{
Marin H Kollef MD
}

\begin{abstract}
Currently, >20 million people in the United States have asthma, and approximately 15 million adults have been diagnosed with COPD, with approximately the same number not yet having been diagnosed with this condition. Moreover, the overall burden of respiratory diseases is still increasing, in part due to environmental factors, such as air pollution. At the same time, the number of patients requiring hospitalization as well as the number of individuals admitted to ICUs from emergency departments has been on the rise over the last decade. Because of the cost to the health-care system, the burden of respiratory diseases, hospitalizations, and ICU admissions also falls on society; it is paid for with tax dollars, higher health insurance rates, and lost productivity. Respiratory therapists (RTs) are in a unique position to influence health-care delivery in a number of settings that include acutely ill hospitalized patients and those with chronic conditions in ambulatory settings. Clinical studies have demonstrated the value of RTs in specific areas, including the performance of medical procedures, the development and implementation of protocols aimed at weaning patients from mechanical ventilation and providing lung-protective ventilation, optimal delivery of in-patient respiratory treatments, the application of disease management programs for COPD, and as part of rapid response teams. However, due to increasing scrutiny of health-care expenditures and limited resources, there is a growing need to document the impact of health-care providers in terms of clinical outcomes. As a profession, RTs should continue to describe the impact they have on patient outcomes and the value they bring to our health-care system. Promoting such investigative outcomes research, along with enhancing the professional aspects of the field of respiratory care, will ensure that the value of RTs does not go unappreciated. Key words: respiratory therapist; professionalism; outcomes; hospital. [Respir Care 2017;62(12):1602-1610. (C) 2017 Daedalus Enterprises]
\end{abstract}

\section{Introduction}

The delivery of health care is becoming increasingly specialized and complex as the understanding of the basic

\footnotetext{
Dr Kollef is affiliated with the Division of Pulmonary and Critical Care Medicine, Washington University School of Medicine, St. Louis, Missouri.
}

This work was supported by the Barnes-Jewish Hospital Foundation. Dr Kollef has disclosed no conflicts of interest.

Dr Kollef presented a version of this paper as the 33rd Philip Kittredge Memorial Lecture at the 63rd AARC Congress, held October 4-7, 2017 in Indianapolis, Indiana.

Correspondence: Marin H Kollef MD, Division of Pulmonary and Critical Care Medicine, Washington University School of Medicine, 4523 pathophysiology of disease processes improves and the development of novel therapeutic interventions accelerates. This paradigm certainly holds true for respiratory disorders and the care of critically ill patients, in which respiratory therapists (RTs) play a pivotal role in terms of medical care delivery. The National Institutes of Health National Heart, Lung, and Blood Institute (NHLBI) estimated that lung diseases, excluding lung cancer, caused approximately 235,000 deaths in 2010 and that in 2010, almost 1 in 6 deaths in children $<1 \mathrm{y}$ of age was due to a lung disease. ${ }^{1}$ Moreover, the NHLBI assessed the eco-

Clayton Avenue, Campus Box 8052, St. Louis, MO 63110. E-mail: kollefm@wustl.edu.

DOI: $10.4187 /$ respcare. 05807 
nomic cost of asthma, COPD, and pneumonia to be $\$ 106$ billion in 2009. ${ }^{1}$ Similarly, Eurostat assessments from the European Union found that in 2013, there were $>401,000$ deaths in the European Union resulting from diseases of the respiratory system, equivalent to $8.0 \%$ of all deaths. ${ }^{2}$ These figures highlight the importance of respiratory disorders and critical illness as the foundation for supporting state-of-the-art respiratory care delivery to both hospitalized and ambulatory patients.

In 2000, James K Stoller MD presented the 27th Donald F Egan Scientific Memorial Lecture at the American Association for Respiratory Care Congress, providing a systematic review examining the overall effectiveness of RTs in delivering respiratory care in the ICU setting, in adult non-ICU in-patient care, and in ambulatory care. ${ }^{3} \mathrm{He}$ concluded that "Notwithstanding the considerable body of available evidence that buttresses RTs' effectiveness in delivering care, additional rigorously designed studies are needed to examine RTs' effectiveness in new roles (eg, geriatric care, pediatric care), in new venues (eg, extended care facilities), and to assure the generalizability of available findings to the broad spectrum of healthcare facilities (eg, academic and community-based facilities alike). Finally, the respiratory therapy community must continue to cultivate and support investigative expertise to assure continued inquiry."3 This year's Phil Kittredge Memorial Lecture will attempt to update Dr Stoller's review and provide specific examples from Barnes-Jewish Hospital highlighting the evidence supporting the value of RTs and highlighting the need for additional investigations in this area.

\section{Procedural Skills}

Tracheal intubation is a common procedure performed in patients developing acute respiratory failure and can be performed by a number of medical specialists, including physicians, nurses, and RTs. A number of studies support the safety of RTs performing tracheal intubation in emergent settings or for elective procedures, with the success rates of RTs performing tracheal intubation being comparable with those of physicians. . $^{4}$ The optimal way to ensure proficient training for RTs in tracheal intubation is unclear but was the subject of a recent survey published by Andrew Miller in Respiratory CARe. ${ }^{8}$ He found that the most common training methods employed to train RTs in tracheal intubation included simulation training (86\%), supervised intubations (84\%), and classroom training (65\%). Classroom training lasted a mean of $4.3 \mathrm{~h}$ with a wide range (1-16 h). The majority of RTs $(91 \%)$ were required to complete 10 or fewer supervised intubations before becoming certified for competency in this procedure. Skill recertification was automatic if a minimum number of endotracheal intubations were performed annually in $78 \%$ of centers, and $11 \%$ required a written test or classroom training annually. The primary barrier cited for RTs not intubating was lack of need. The latter finding is important and may explain the observation that RT performance of tracheal intubation usually occurs in smaller hospitals, when physician providers are not available, or in field conditions. Nevertheless, the literature suggests that RTs can carry out this procedure proficiently when properly trained.

Similar to tracheal intubation, fiberoptic bronchoscopy is a common procedure performed in patients with respiratory disorders. At Barnes-Jewish Hospital, experienced RTs have played an important role on the bronchoscopy service, as well as on the interventional pulmonary service. RTs provide important assistance to the physicians performing bronchoscopy in terms of providing topical sedation, securing and maintaining the patient's airway, assisting in carrying out endobronchial and transbronchial biopsies with forceps, and training fellows and novice bronchoscopists on the anatomy of the airways and proper technique in carrying out fiberoptic bronchoscopy. Coppolo et $\mathrm{al}^{9}$ reviewed the role of physician-RT teams in carrying out bronchoscopic procedures and demonstrated that RTs can provide high-quality, low-cost, safe bronchoscopic assistance to pulmonary specialists, including performing biopsies.

In addition to fiberoptic bronchoscopy, RTs more regularly are called upon to carry out bronchoalveolar lavage (BAL) using various blindly positioned telescoping tubes. At Barnes-Jewish Hospital, we demonstrated the safety of the mini-BAL procedure for the evaluation of suspected ventilator-associated pneumonia carried out by RTs. ${ }^{10}$ Comparing mini-BAL performed by RTs with physician-directed protected specimen brush deployment with a fiberoptic bronchoscope, there was no change in arterial blood oxygen saturation or heart rate that occurred after mini-BAL. Moreover, there was good diagnostic agreement for quantitative cultures obtained with the protected specimen brush and mini-BAL (kappa statistic, 0.63; concordance, $83.3 \%$ ). RTs performing mini-BAL may provide respiratory cultures from intubated patients before changing or starting antibiotics, allowing for the collection of lower respiratory specimens that are more likely to demonstrate the presence of pathogenic bacteria. ${ }^{11}$

Taken together, these findings suggest that there are a number of procedures currently performed by other healthcare providers that could be carried out by RTs. Future research demonstrating the safety of RTs carrying out specific types of procedures (eg, fiberoptic bronchoscopy for the removal of impacted secretions, bedside ultrasonography to assess the presence of pleural effusions and consolidation), along with potential cost savings implications, are needed to advance this field. 


\section{Protocol/Guideline Development and Optimization}

\section{Weaning Mechanical Ventilation}

Mechanical ventilation is one of the most common forms of medical therapy administered within ICUs. Weaning patients from mechanical ventilation has been referred to as part of the "bread and butter" of respiratory care practices provided by physicians, RTs, and nurses working in ICUs, post-anesthesia recovery units, intermediate care units, and other specialized hospital settings. ${ }^{12}$ However, the procedural methodology or precedent for weaning or liberating patients from mechanical ventilation has historically been physician-guided weaning. Despite a general consensus on what weaning represents, continued controversy exists on how it should best be achieved. ${ }^{13}$ Most of the earlier randomized, controlled trials focused on identifying the best ventilator withdrawal technique for weaning patients from mechanical ventilation. ${ }^{14,15}$ However, the more central issue with regard to optimizing the weaning process is how we can best identify patients who are ready to be liberated from mechanical ventilation at the earliest point in time.

At Barnes-Jewish Hospital, we carried out a randomized controlled trial comparing the practice of protocol-directed weaning from mechanical ventilation implemented by nurses and RTs with traditional physician-directed weaning. ${ }^{16}$ We enrolled 357 subjects in the study, with the primary outcome being the duration of mechanical ventilation from tracheal intubation until discontinuation of mechanical ventilation. The median duration of mechanical ventilation was $35 \mathrm{~h}$ for the protocol-directed group (first quartile, $15 \mathrm{~h}$; third quartile, $114 \mathrm{~h}$ ) compared with $44 \mathrm{~h}$ for the physician-directed group (first quartile, $21 \mathrm{~h}$; third quartile, 209 h). Kaplan-Meier analysis demonstrated that subjects randomized to protocol-directed weaning had significantly shorter durations of mechanical ventilation compared with subjects randomized to physiciandirected weaning (chi-square $=3.62, P=.057, \log$ rank test; chi-square $=5.12, P=.02$, Wilcoxon test). Cox proportional-hazards regression analysis, adjusting for other covariates, showed that the rate of successful weaning was significantly greater for subjects receiving protocol-directed weaning compared with subjects receiving physician-directed weaning (risk ratio 1.31, 95\% CI $1.15-1.50, P=.039)$. The hospital mortality rates for the 2 treatment groups were similar $(22.3 \%$ for protocol-directed vs $23.6 \%$ for physician-directed, $P=.78$ ). Hospital cost savings for subjects in the protocol-directed group were $\$ 42,960$ compared with hospital costs for subjects in the physician-directed group. Protocol-guided weaning of mechanical ventilation, as performed by nurses and RTs, was safe and led to extubation more rapidly than physician-directed weaning.
Moreover, based on this experience, all hospitals in the BJC HealthCare system (12 hospitals and a rehabilitation center) require that protocolized weaning of mechanical ventilation occur in all units caring for patients with respiratory failure.

A consensus conference reinforced the ability of protocol-guided weaning carried out by RTs or nurses to be a successful method for allowing timely weaning from mechanical ventilation. ${ }^{17}$ For patients who pass a spontaneous breathing trial (SBT), the decision to extubate should still be guided by clinical judgment and objective data to minimize the risk of unnecessary reintubations and selfextubations. Additionally, the protocols employed for weaning should not represent rigid rules but, rather, guides to patient care. Moreover, these protocols should evolve over time as clinical and institutional experience with them increases and new research becomes available. The most useful protocols aim to safely and efficiently liberate patients from mechanical ventilation, reducing unnecessary or harmful variations in approach. Given the close interaction of RTs with mechanically ventilated patients, they are in an ideal position to help develop and facilitate the successful implementation of weaning protocols.

More recently, the Centers for Disease Control and Prevention (CDC) introduced ventilator-associated event definitions in January 2013. ${ }^{18}$ To this day, little is known about ventilator-associated event prevention and whether prevention of ventilator-associated events impacts other important clinical outcomes, such as hospital mortality and lengths of stay. However, investigators supported by the CDC hypothesized that daily, protocolized spontaneous awakening trials and SBTs carried out by nurses and RTs, respectively, might prevent ventilator-associated events. ${ }^{19}$ They carried out a prospective study in 12 community ICUs employing an opt-out protocol for nurses and RTs to perform paired daily spontaneous awakening trials and SBTs. Eight other community ICUs served as controls and conducted surveillance alone. They tracked 5,164 consecutive episodes of mechanical ventilation: 3,425 in collaborative units and 1,739 in surveillance-only units. Within the collaborative units, significant increases in spontaneous awakening trials, SBTs, and percentage of SBTs performed without sedation were mirrored by significant decreases in duration of mechanical ventilation and length of hospital stay. There was no change in ventilator-associated event risk per ventilator day, but significant decreases in ventilator-associated event risk per episode of mechanical ventilation were observed. Within surveillanceonly units, there were no significant changes in spontaneous awakening trials, SBTs, or ventilator-associated event rates. Although it is still unclear whether ventilator-associated events represent a true marker of medical quality in mechanically ventilated patients, this multi-center study confirmed the importance of protocolized weaning of mechanical ventilation 


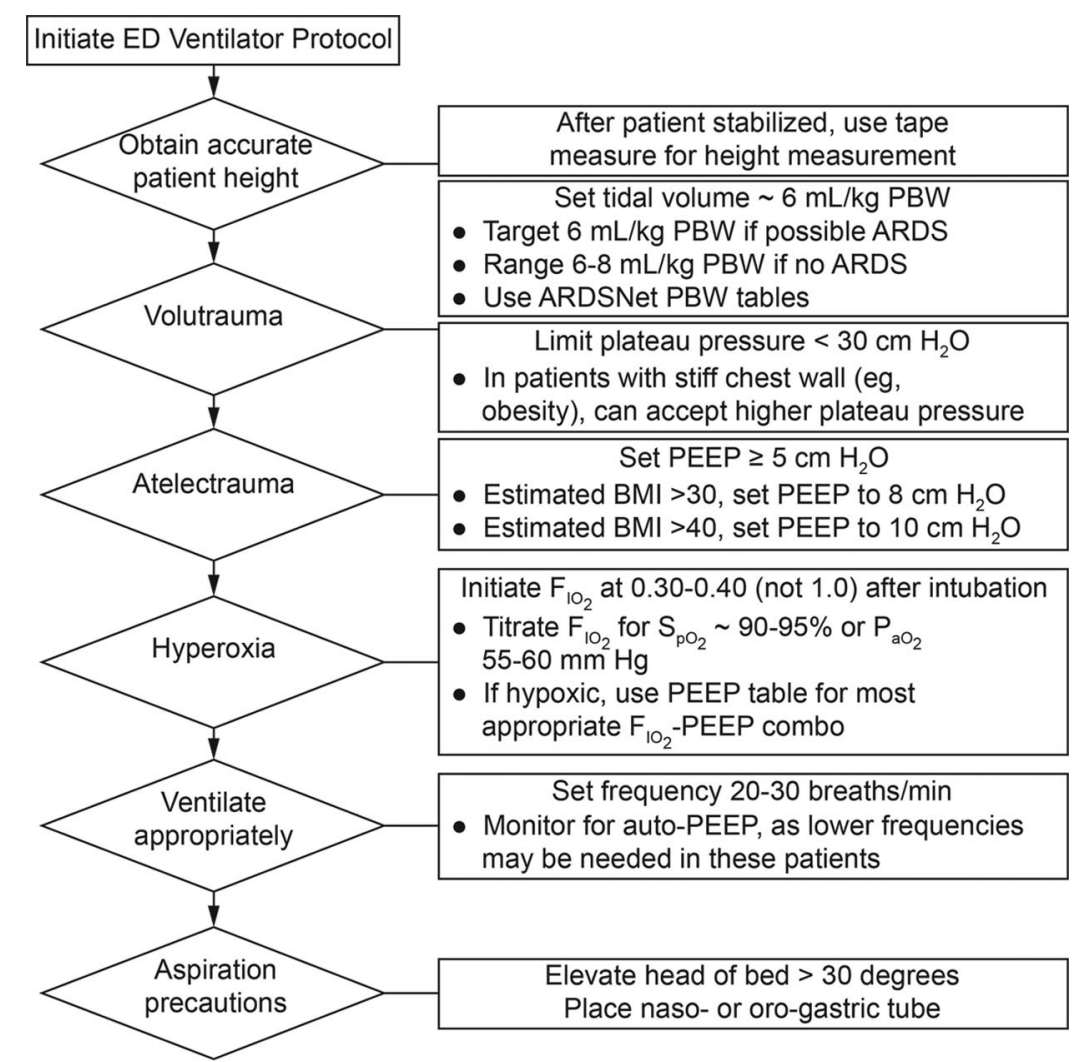

Fig. 1. Emergency department (ED) ventilator protocol. PBW = predicted body weight. BMI = body mass index.

carried out by RTs to decrease the duration of mechanical ventilation.

\section{Lung-Protective Ventilation}

Minimizing iatrogenic lung injury is recognized as a key element of the application of mechanical ventilation for patients with respiratory failure. ${ }^{20}$ The primary strategy for achieving this goal is to avoid overstretch of the lung with the appropriate selection of tidal volume and PEEP. At Barnes-Jewish Hospital, we evaluated the efficacy of an emergency department-based lung-protective mechanical ventilation protocol for the prevention of pulmonary complications (Fig. 1). ${ }^{21}$ This protocol was developed in conjunction with the hospital's Department of Respiratory Therapy and was directly implemented by RTs in the emergency department setting. The protocol that the RTs employed is provided in Figure 1.

We carried out a quasi-experimental, before-after study that consisted of a preintervention period, a run-in period of approximately 6 months, and a prospective intervention period. The intervention was a multifaceted emergency department-based mechanical ventilator protocol carried out by RTs targeting lung-protective tidal volume, appropriate setting of PEEP, rapid oxygen weaning, and head- of-bed elevation. A propensity score-matched analysis was used to evaluate the primary outcome, which was the composite incidence of ARDS and ventilator-associated events. A total of 1,192 subjects in the pre-intervention group and 513 subjects in the intervention group were included. Lungprotective ventilation increased by $48.4 \%$ in the intervention group. In the propensity score-matched analysis ( $n=490$ in each group), the primary outcome occurred in 71 subjects $(14.5 \%)$ in the preintervention group compared with 36 subjects $(7.4 \%)$ in the intervention group (adjusted odds ratio $0.47,95 \% \mathrm{CI} 0.31-0.71$ ). There was an increase in ventilator-free days (mean difference $3.7,95 \%$ CI $2.3-$ 5.1), ICU-free days (mean difference $2.4,95 \%$ CI 1.0 3.7 ), and hospital-free days (mean difference $2.4,95 \% \mathrm{CI}$ 1.2-3.6) associated with the intervention. The mortality rate was $34.1 \%$ in the preintervention group and $19.6 \%$ in the intervention group (adjusted odds ratio 0.47, 95\% CI 0.35-0.63). This experience for the first time suggested that implementation of a mechanical ventilator protocol in the emergency department by RTs is feasible and could significantly improve the delivery of safe mechanical ventilation and clinical outcomes, including mortality.

As part of the same quality improvement initiative, we also assessed the impact of having RTs implement a lung- 
protective strategy of ventilation in the emergency department as a means of improving the outcome of patients with established ARDS at the time of emergency department presentation. ${ }^{22}$ Using the same lung-protective ventilator protocol described above, a total of 229 subjects (186 preintervention group, 43 intervention group) were studied in the emergency department of Barnes-Jewish Hospital. The intervention was associated with significant changes $(P<.01$ for all $)$ in tidal volume, PEEP, breathing frequency, oxygen administration, and head-of-bed elevation. There was a reduction in median (interquartile range) emergency department tidal volume from 8.1 (7.0-9.1) to $6.4(6.1-6.7) \mathrm{mL} / \mathrm{kg}$ predicted body weight and an increase in lung-protective ventilation from 11.1 to $61.5 \%$ $(P<.01)$. The intervention was associated with a reduction in mortality from 54.8 to $39.5 \%$ (odds ratio 0.38 , 95\% CI 0.17-0.83, $P=.02$ ) and a 3.9-d increase in ventilator-free days $(P<.01)$. This study of mechanically ventilated subjects with ARDS demonstrated that RTs implementing a mechanical ventilator protocol in the emergency department could improve the clinical outcomes of these patients by minimizing the potential injurious influence of mechanical ventilation.

The increased recognition of improved outcomes in patients without ARDS undergoing mechanical ventilation employing ventilator delivery strategies aimed at minimizing driving pressure and optimizing lung mechanics reinforces the need for the general application of these principles. ${ }^{23,24}$ RTs again are in a unique position to implement protocols in their institutions to see that this occurs as well as to conduct further research into how to generalize the implementation of these approaches in non-teaching hospitals and other types of facilities (eg, rehabilitation centers, long-term acute care settings, chronic ventilator hospitals).

\section{In-Patient RT Treatments}

The administration of respiratory treatments is a necessary component of the care of hospitalized patients, especially those individuals with thoracoabdominal disease processes. However, a number of clinical studies suggest that respiratory treatments are often needlessly prescribed to patients who do not derive benefit from their administration. ${ }^{25-34}$ Few investigations have attempted to directly define the optimal method for the utilization of respiratory treatments or to determine the impact of various respiratory care utilization strategies on patient outcomes ${ }^{35-37}$ This represents an important area of investigation because of the potential complications associated with inappropriate use of respiratory treatments and the need to conserve valuable medical resources for treatments that are more likely to provide patient benefits. ${ }^{38,39}$
We carried out a prospective study at Barnes-Jewish Hospital to compare the clinical outcomes of subjects receiving respiratory treatments by RTs employing a respiratory care protocol with physician-directed respiratory treatment orders. ${ }^{40}$ Six hundred ninety-four consecutive hospitalized non-ICU subjects ordered to receive respiratory treatments were evaluated according to the medical firm they were admitted to (firm A, intervention group; firms B and C, control groups). Discordant respiratory care orders, respiratory care charges, hospital stay, and patient-specific complications were assessed as the primary outcomes. Discordant orders were defined as written orders for respiratory treatments that were not clinically indicated as well as orders omitting treatments that were clinically indicated according to the hospital's protocolbased treatment algorithms. Firm A subjects $(n=239)$ received RT-directed treatments and had a statistically lower rate of discordant respiratory care orders $(24.3 \%)$ as compared with subjects receiving physician-directed treatments in firms $\mathrm{B}(n=205 ; 58.5 \%)$ and $\mathrm{C}(n=250$; $56.8 \% ; P<.001)$. No statistically significant differences in patient complications were observed. The average numbers of respiratory treatments and respiratory care charges were statistically lower for firm A subjects $(10.7 \pm 13.7$ treatments; $\$ 868 \pm \$ 1,519)$ as compared with subjects in firms B (12.4 \pm 12.7 treatments, $\$ 1,124 \pm \$ 1,339)$ and C $(12.3 \pm 13.4$ treatments, $\$ 1,054 \pm \$ 1,346, P=.009$ [treatments] and $P<.001$ [respiratory care charges]). Additionally, discordant respiratory treatment orders were associated with significantly greater charges compared with concordant respiratory treatment orders (Fig. 2). This experience, similar to that described by Stoller et al, ${ }^{37}$ highlights the ability of RTs to recommend respiratory treatments that are consistent with hospital-based protocols and consensus guidelines more often than physician-directed respiratory treatments. Additionally, these studies suggest that RTs may be in a better position to triage respiratory treatments, especially during times when RT resources are limited or the need for such treatments increases, such as with influenza outbreaks.

\section{Out-Patient COPD Management}

Patients with COPD make up one of the most frequent disease states interacting with RTs and often require frequent hospital admissions and/or visits to the emergency department for exacerbations of their lung disease. ${ }^{41,42}$ Such readmissions increase health-care costs and expose COPD patients to the added risks of hospital readmission, including nosocomial infections and increased risk of mortality. ${ }^{43}$ It is common for many of these repeat visits to occur within 30 $180 \mathrm{~d}$ following hospital discharge for a COPD exacerbation. ${ }^{43,44}$ Disease management is a term that can be broadly defined as a comprehensive strategy for improving overall 


\section{Evaluating the Value of the Respiratory Therapist}

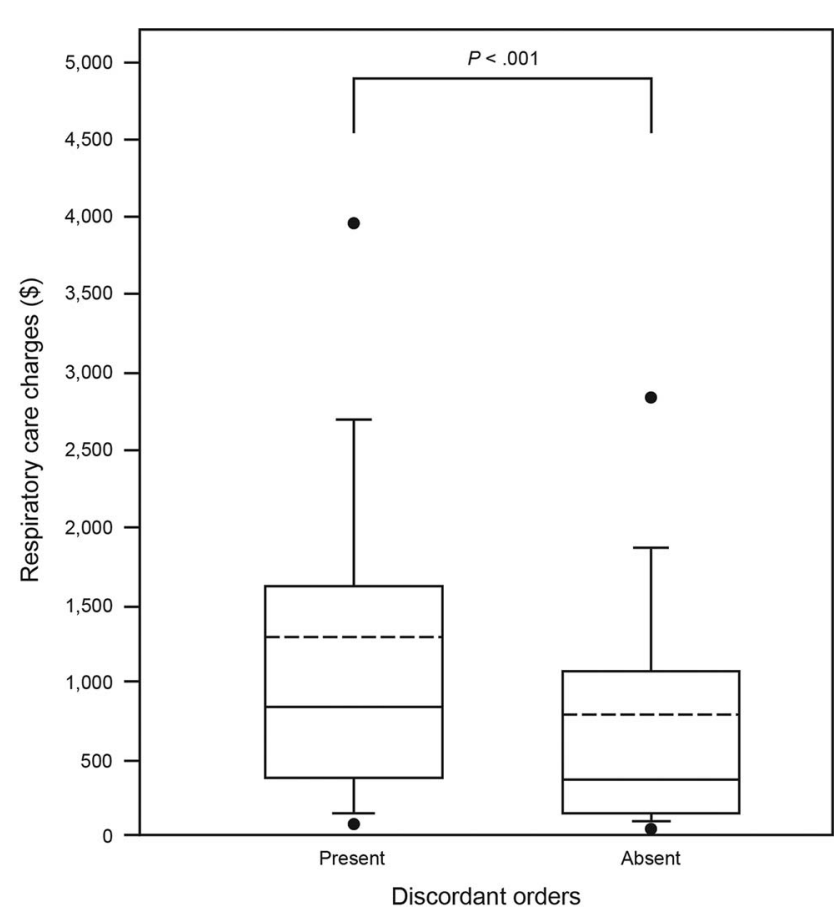

Fig. 2. Box plots for respiratory care charges in patients with and without discordant respiratory care orders. Boxes represent the 25th to 75th percentiles, with the 50th percentile (solid line) and mean (broken line) values within the boxes. The 10th and 90th percentiles are shown as bars, and points mark the 5th and 95th percentiles.

health status and reducing health-care costs in chronic conditions. ${ }^{45}$ These programs are often conducted by physician extenders and may include education about the underlying disease, optimization of evidence-based medications, support from case managers, and institution of self-management principles. ${ }^{46}$ COPD represents a unique chronic respiratory disorder for which RT disease management transition teams could be utilized to facilitate the hospital discharge of patients with COPD to the out-patient setting and to potentially prevent hospital readmissions.

Disease management programs for chronic medical conditions, such as congestive heart failure and diabetes mellitus, have been successfully implemented. ${ }^{47,48}$ Disease management programs for COPD have also been studied with varied success. ${ }^{49,50}$ We performed a study to determine whether a resource-intensive RT disease management program could reduce rehospitalization and emergency department visits for subjects hospitalized with an exacerbation of COPD. ${ }^{51}$ We enrolled 428 subjects (214 intervention, 214 control) in a prospective trial comparing the RT disease management program with usual care. The primary outcome (combined non-hospitalized emergency department visits and hospital readmissions for a COPD exacerbation during the 6-month follow-up) was similar for the 2 study groups (94 vs $159, P=.08$ ). When the individual components of the primary end point were an- alyzed individually, the percentage of subjects with nonhospitalized emergency department visits for COPD exacerbations was similar between groups $(15.0 \%$ vs $15.9 \%$, $P=$.79). However, hospital readmission for a COPD exacerbation was significantly lower in the intervention group $(20.1 \%$ vs $28.5 \%, P=.042)$. The median (interquartile range) duration of hospitalization for a COPD exacerbation was significantly less for the intervention group (5 [3-11] d vs 8 [4-18.5] d, $P=.045)$. Additionally, in-patient hospital days (306 d vs $523 \mathrm{~d}, P=.02$ ) and ICU days ( $17 \mathrm{~d}$ vs $53 \mathrm{~d}, P=.02$ ) due to COPD exacerbations were significantly lower for the intervention group. Our RT disease management program, although requiring substantial time and RT resources, was associated with reduced hospital readmission, fewer ICU days, and shorter hospital stays due to COPD exacerbations. Given the increasing scrutiny hospitals face over early hospital readmissions from payers, utilization of an RT disease management team for patients with COPD could be considered helpful in reducing the number of such readmissions.

\section{RTs and Rapid Response Teams}

Rapid response teams have been introduced into hospitals worldwide in an effort to improve the outcomes of deteriorating hospitalized patients. ${ }^{52}$ There is considerable variability in the makeup of rapid response teams, which often include physician extenders, such as nurses and RTs, especially in the United States. The overall impact of rapid response teams on patient outcomes has been mixed with a call for more comprehensive research in this area. ${ }^{53-56}$

Development of the rapid response team at Barnes-Jewish Hospital began in 2005 and initially targeted the 8 general medicine units within the hospital. This included formalizing the rapid response team and providing staff education on how to activate and utilize it. The rapid response team is made up of an ICU nurse and an RT, with a senior medical resident being available as well. Rapid response team activations between 2006 and 2008 were initiated by the nursing staff on the general medicine units as part of routine nursing practice. Starting in 2009, rapid response team activations could be initiated by the nursing staff as well as by automated clinical deterioration alerts generated by a hospital-based algorithm. The development of the automated clinical deterioration alerts was part of a collaborative program carried out between Washington University and Barnes-Jewish Hospital. ${ }^{57-60}$ Patients identified as at risk for clinical deterioration with the automated clinical deterioration alerts algorithm had an automated alert sent in real time to the rapid response team. Automated clinical deterioration alerts are generated $24 \mathrm{~h} / \mathrm{d}$ $7 \mathrm{~d} /$ week.

Introduction of the rapid response team occurred in 2006, with the staged addition of the automated clinical deteri- 


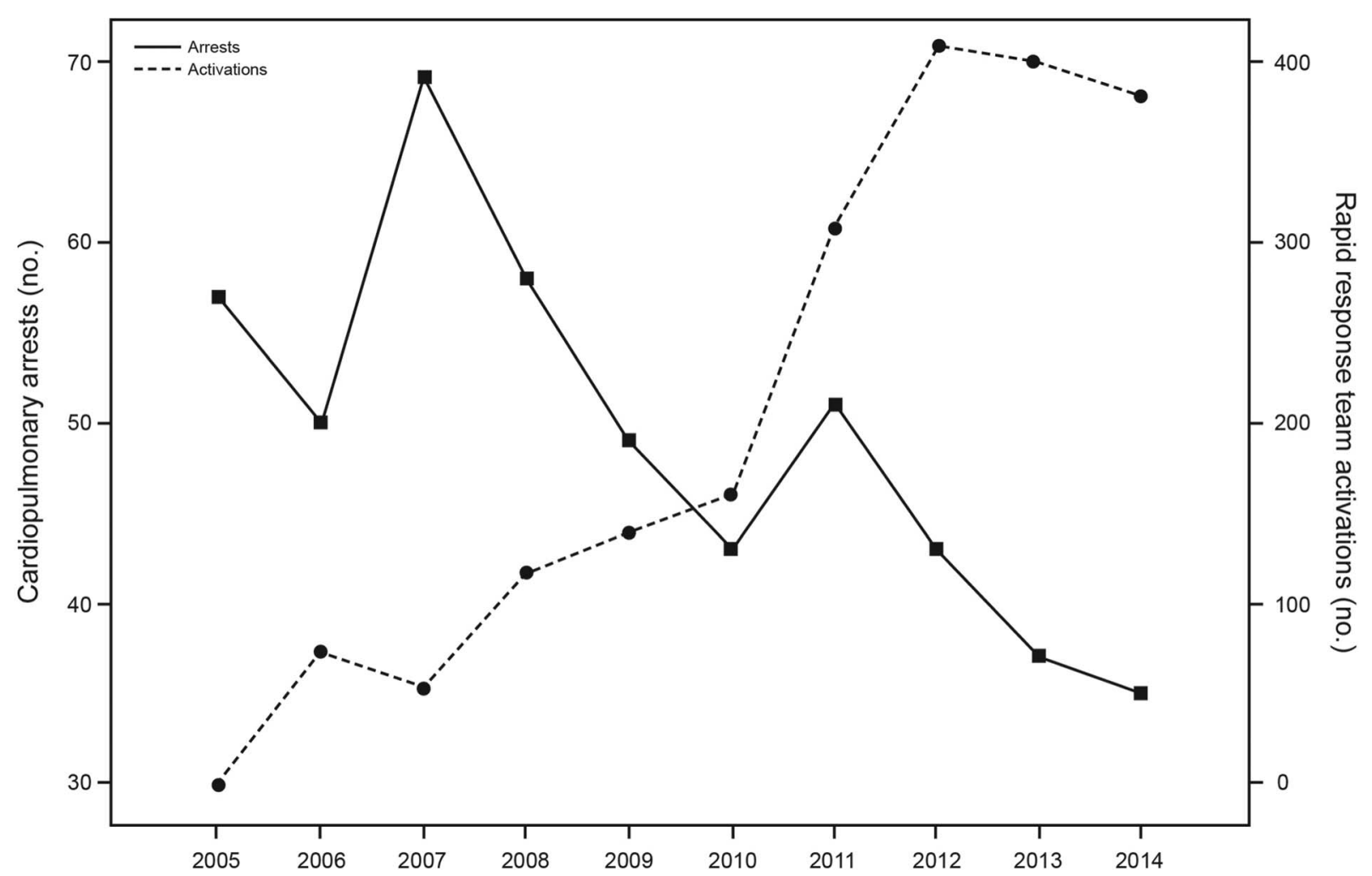

Fig. 3. Cardiopulmonary arrests and rapid response team activations between January 1, 2005 and December 31, 2014 for the 8 general medicine units.

oration alerts in 2009. Statistically significant year-to-year decreases in mortality were observed after the introduction of the rapid response team through 2014. ${ }^{61}$ Similarly, yearto-year decreases in the number of cardiopulmonary arrests and median lengths of stay were observed. There was a statistically significant year-to-year increase in the number of rapid response team activations for these units that was inversely correlated with the occurrence of cardiopulmonary arrests (Fig. 3). This single-institution experience suggests that since the introduction of an rapid response team, which included RTs as team members, lower hospital mortality, fewer cardiopulmonary arrests, and shorter hospital stays have been observed on the medicine units employing the rapid response teams.

\section{Conclusions}

The experience at Barnes-Jewish Hospital, along with that at other centers, suggests that RTs play a pivotal role in the care and management of complex patients with respiratory diseases. However, there is also recent evidence suggesting that the profession of respiratory care is at risk of losing practitioners due in part to lack of opportunities for professional growth and contracted scope of clinical practice in some settings. ${ }^{62}$ It is imperative that the value of RTs in terms of providing medical services to patients with acute and chronic conditions, both within the hospital and in the out-patient setting, be demonstrated and documented in well-thought out observational and interventional investigations. Only in this way can we continue to advance the profession of respiratory care and expand its footprint in this era of advancing and increasingly complex medical therapies.

\section{REFERENCES}

1. National Heart, Lung and Blood Institute. Disease Statistics. https:// www.nhlbi.nih.gov/about/documents/factbook/2012/chapter4. Accessed August 30, 2017.

2. Eurostat. Respiratory disease statistics. http://ec.europa.eu/eurostat/ statistics-explained/index.php/Respiratory_diseases_statistics. Accessed August 30, 2017.

3. Stoller JK. Are respiratory therapist effective? Assessing the evidence. Respir Care 2001;46(1):56-66.

4. Adams K, Scott R, Perkin RM, Langga L. Comparison of intubation skills between interfacility transport team members. Pediatr Emerg Care 2000;16(1):5-8.

5. Noblett KE, Meibalane R. Respiratory care practitioners as primary providers of neonatal intubation in a community hospital: an analysis. Respir Care 1995;40(10):1063-1067.

6. Thalman JJ, Rinaldo-Gallo S, MacIntyre NR. Analysis of an endotracheal intubation service provided by respiratory care practitioners. Respir Care 1993;38(5):469-473.

7. Zyla EL, Carlson J. Respiratory care practitioners as secondary providers of endotracheal intubation: one hospital's experience. Respir Care 1994;39(1):30-33. 


\section{Evaluating the Value of the ResPiratory Therapist}

8. Miller AG. Endotracheal intubation training and skill maintenance for respiratory therapists. Respir Care 2017;62(2):156-162.

9. Coppolo DP, Brienza LT, Pratt DS, May JJ. A role for the respiratory therapist in flexible fiberoptic bronchoscopy. Respir Care 1985;30(5): 323-327.

10. Kollef MH, Ward S. The influence of mini-BAL cultures on patient outcomes: implications for the antibiotic management of ventilatorassociated pneumonia. Chest 1998;113(2):412-420.

11. Fagon JY, Chastre J, Rouby JJ. Is bronchoalveolar lavage with quantitative cultures a useful tool for diagnosing ventilator-associated pneumonia? Crit Care 2007;11(2):123.

12. Pierson DJ. Weaning from mechanical ventilation: why all the confusion? Respir Care 1995;40(3):228-232.

13. Chatburn RL, Deem S. Respiratory controversies in the critical care setting: should weaning protocols be used with all patients who receive mechanical ventilation? Respir Care 2007;52(5):609-619; discussion 619-621.

14. Brochard L, Rauss A, Benito S, Conti G, Mancebo J, Rekik N, et al. Comparison of three methods of gradual withdrawal from ventilatory support during weaning from mechanical ventilation. Am J Respir Crit Care Med 1994;150(4):896-903.

15. Esteban A, Frutos F, Tobin MJ, Alía I, Solsona JF, Valverdú I, et al. A comparison of four methods of weaning patients from mechanical ventilation. N Engl J Med 1995;332(6):345-350.

16. Kollef MH, Shapiro SD, Silver P, St John RE, Prentice D, Sauer S, et al. A randomized, controlled trial of protocol-directed versus physician-directed weaning from mechanical ventilation. Crit Care Med 1997;25(4):567-574.

17. Ely EW, Meade MO, Haponik EF, Kollef MH, Cook DJ, Guyatt GH, Stoller JK. Mechanical ventilator weaning protocols driven by nonphysician health-care professionals: evidence-based clinical practice guidelines. Chest 2001;120(6 Suppl):454S-463S.

18. Magill SS, Klompas M, Balk R, Burns SM, Deutschman CS, Diekema $\mathrm{D}$, et al. Developing a new, national approach to surveillance for ventilator-associated events: executive summary. Clin Infect Dis 2013;57(12):1742-1746.

19. Klompas M, Anderson D, Trick W, Babcock H, Kerlin MP, Li L, et al. The preventability of ventilator-associated events: the CDC Prevention Epicenters Wake Up and Breathe Collaborative. Am J Respir Crit Care Med 2015;191(3):292-301.

20. Henderson WR, Chen L, Amato MB, Brochard LJ. Fifty years of research in ARDS: respiratory mechanics in acute respiratory distress syndrome. Am J Respir Crit Care Med 2017 [Epub ahead of print]. doi: 10.1164/rccm.201612-2495CI.

21. Fuller BM, Ferguson IT, Mohr NM, Drewry AM, Palmer C, Wessman BT, et al. Lung-protective ventilation initiated in the emergency department (LOV-ED): a quasi-experimental, before-after trial. Ann Emerg Med 2017;70(3):406-418.

22. Fuller BM, Ferguson IT, Mohr NM, Drewry AM, Palmer C, Wessman BT, et al. A quasi-experimental, before-after trial examining the impact of an emergency department mechanical ventilator protocol on clinical outcomes and lung-protective ventilation in acute respiratory distress syndrome. Crit Care Med 2017;45(4):645-652.

23. Neto AS, Hemmes SN, Barbas CS, Beiderlinden M, FernandezBustamante A, Futier E, et al. Association between driving pressure and development of postoperative pulmonary complications in patients undergoing mechanical ventilation for general anaesthesia: a meta-analysis of individual patient data. Lancet Respir Med 2016; 4(4):272-280.

24. Guo L, Wang W, Zhao N, Guo L, Chi C, Hou W, et al. Mechanical ventilation strategies for intensive care unit patients without acute lung injury or acute respiratory distress syndrome: a systematic review and network meta-analysis. Crit Care 2016;20(1):226.
25. Kollef MH. Outcomes research as a tool for defining the role of respiratory care practitioners in the ICU setting. New Horiz 1998; 6(1):91-98

26. Zibrak JD, Rossetti P, Wood E. Effect of reductions in respiratory therapy on patient outcomes. N Engl J Med 1986;315(5):292-295.

27. Celli BR, Rodriguez KS, Snider GL. A controlled trial of intermittent positive pressure breathing, incentive spirometry, and deep breathing exercises in preventing pulmonary complications after abdominal surgery. Am Rev Respir Dis 1984;130(1):12-15.

28. Johnson D, Kelm C, To T, Hurst T, Naik C, Gulka I, et al. Postoperative physical therapy after coronary artery bypass surgery. Am J Respir Crit Care Med 1995;152(3):953-958.

29. Hall JC, Tarala RA, Tapper J, Hall JL. Prevention of respiratory complications after abdominal surgery: a randomized clinical trial. BMJ 1996;312(7024):148-152; discussion 152-153.

30. Alexander E, Weingarten S, Mohsenifar Z. Clinical strategies to reduce utilization of chest physiotherapy without compromising patient care. Chest 1996;110(2):430-432.

31. Dhand R, Jubran A, Tobin MJ. Bronchodilation delivery by metereddose inhaler in ventilation-supported patients. Am J Respir Crit Care Med 1995;151(6):1827-1833.

32. Idris AH, McDermott MF, Raucci JC, Morrabel A, McGorray S, Hendeles L. Emergency department treatment of severe asthma: metered-dose inhaler plus holding chamber is equivalent in effectiveness to nebulizer. Chest 1993;103(3):665-672.

33. Colacone A, Afilalo M, Wolkove N, Kreisman H. A comparison of albuterol administered by metered dose inhaler (and holding chamber) or wet nebulizer in acute asthma. Chest 1993;104(3):835-841.

34. Pilon CS, Leathley M, London R, McLean S, Phang PT, Priestley R, et al. Practice guideline for arterial blood gas measurement in the intensive care unit decreases numbers and increases appropriateness of tests Crit Care Med 1997;25(8):1308-1313.

35. Kirby EG, Durbin CG Jr. Establishment of a respiratory assessment team is associated with decreased mortality in patients re-admitted to the ICU. Respir Care 1996;41:903-907.

36. Stoller JK, Skibinski CI, Giles DK, Kester EL, Haney DJ. Physicianordered respiratory care vs physician-ordered use of a respiratory therapy consult service: results of a prospective observational study. Chest 1996;110(2):422-429.

37. Stoller JK, Mascha EJ, Kester L, Haney D. Randomized controlled trial of physician-directed versus respiratory therapy consult servicedirected respiratory care to adult non-ICU inpatients. Am J Respir Crit Care Med 1998;158(4):1068-1075.

38. Connors AF Jr, Hammon WE, Martin RJ, Rogers RM. Chest physical therapy: the immediate effect on oxygenation in acutely ill patients. Chest 1980;78(4):559-564

39. Kollef MH. Prolonged use of ventilator circuits and ventilator-associated pneumonia: a model for identifying the optimal clinical practice Chest 1998;113(2):267-269.

40. Kollef MH, Shapiro SD, Clinkscale D, Cracchiolo L, Clayton D, Wilner R, Hossin L. The effect of respiratory therapist-initiated treatment protocols on patient outcomes and resource utilization. Chest 2000;117(2):467-475.

41. Hunter LC, Lee RJ, Butcher I, Weir CJ, Fischbacher CM, McAllister D, et al. Patient characteristics associated with risk of first hospital admission and readmission for acute exacerbation of chronic obstructive pulmonary disease (COPD) following primary care COPD diagnosis: a cohort study using linked electronic patient records. BMJ Open 2016;6(1):e009121.

42. Alexopoulos EC, Malli F, Mitsiki E, Bania EG, Varounis C, Gourgoulianis KI. Frequency and risk factors of COPD exacerbations and hospitalizations: a nationwide study in Greece. Int J Chron Obstruct Pulmon Dis 2015;10:2665-2674. 
43. Hartl S, Lopez-Campos JL, Pozo-Rodriguez F, Castro-Acosta A, Studnicka M, Kaiser B, Roberts CM. Risk of death and readmission of hospital-admitted COPD exacerbations: European COPD Audit. Eur Respir J 2016;47(1):113-121.

44. Ford ES. Hospital discharges, readmissions, and ED visits for COPD or bronchiectasis among US adults: findings from the nationwide inpatient sample 2001-2012 and Nationwide Emergency Department Sample 2006-2011. Chest 2015;147(4):989-998.

45. Martínez-González NA, Berchtold P, Ullman K, Busato A, Egger M. Integrated care programmes for adults with chronic conditions: a meta-review. Int J Qual Health Care 2014;26(5):561-570.

46. Shaw RJ, McDuffie JR, Hendrix CC, Edie A, Lindsey-Davis L, Nagi A, et al. Effects of nurse-managed protocols in the outpatient management of adults with chronic conditions: a systematic review and meta-analysis. Ann Intern Med 2014;161(2):113-121.

47. Glasgow RE, Funnell MM, Bonomi AE, Davis C, Beckham V, Wagner EH. Self-management aspects of the improving chronic illness care breakthrough series: implementation with diabetes and heart failure teams. Ann Behav Med 2002;24(2):80-87.

48. Ofman JJ, Badamgarav E, Henning JM, Knight K, Gano AD Jr, Levan RK, et al. Does disease management improve clinical and economic outcomes in patients with chronic diseases? a systematic review. Am J Med 2004;117(3):182-192.

49. Rice KL, Dewan N, Bloomfield HE, Grill J, Schult TM, Nelson DB, et al. Disease management program for chronic obstructive pulmonary disease: a randomized controlled trial. Am J Respir Crit Care Med 2010;182(7):890-896.

50. Fan VS, Gaziano JM, Lew R, Bourbeau J, Adams SG, Leatherman $\mathrm{S}$, et al. A comprehensive care management program to prevent chronic obstructive pulmonary disease hospitalizations: a randomized, controlled trial. Ann Intern Med 2012;156(10):673-683.

51. Silver PC, Kollef MH, Clinkscale D, Watts P, Kidder R, Eads B, et al. A respiratory therapist disease management program for subjects hospitalized with COPD. Respir Care 2017;62(1):1-9.
52. Jones D, Holmes J, Currey J, Fugaccia E, Psirides AJ, Singh MY, et al. Proceedings of the 12th International Conference on Rapid Response Systems and Medical Emergency Teams. Anaesth Intensive Care 2017;45(4):511-517.

53. Chan PS, Khalid A, Longmore LS, Berg RA, Kosiborod M, Spertus JA. Hospital-wide code rates and mortality before and after implementation of a rapid response team. JAMA 2008;300(21):2506-2513.

54. Bonafide CP, Localio AR, Roberts KE, Nadkarni VM, Weirich CM, Keren R. Impact of rapid response system implementation on critical deterioration events in children. JAMA Pediatr 2014;168(1):25-33.

55. Jones DA, DeVita MA, Bellomo R. Rapid-response teams. N Engl J Med 2011;365(2):139-146.

56. Tirkkonen J, Tamminen T, Skrifvars MB. Outcome of adult patients attended by rapid response teams: a systematic review of the literature. Resuscitation 2017;112:43-52.

57. Thiel SW, Rosini JM, Shannon W, Doherty JA, Micek ST, Kollef MH. Early prediction of septic shock in hospitalized patients. J Hosp Med 2010;5(1):19-25.

58. Sawyer AM, Deal EN, Labelle AJ, Witt C, Thiel SW, Heard K, et al. Implementation of a real-time computerized sepsis alert in nonintensive care unit patients. Crit Care Med 2011;39(3):469-473.

59. Bailey TC, Chen Y, Mao Y, Lu C, Hackmann G, Micek ST, et al. A trial of a real-time alert for clinical deterioration in patients hospitalized on general medical wards. J Hosp Med 2013;8(5):236-242.

60. Kollef MH, Chen Y, Heard K, LaRossa GN, Lu C, Martin NR, et al. A randomized trial of real-time automated clinical deterioration alerts sent to a rapid response team. J Hosp Med 2014;9(7):424-429.

61. Kollef MH, Heard K, Chen Y, Lu C, Martin N, Bailey T. Mortality and length of stay trends following implementation of a rapid response system and real-time automated clinical deterioration alerts. Am J Med Qual 2017;32(1):12-18.

62. Smith SG, Endee LM, Benz Scott LA, Linden PL. The future of respiratory care: results of a new york state survey of respiratory therapists. Respir Care 2017;62(3):279-287. 